\title{
COUSIN COMPLEXES AND GENERALIZED FRACTIONS
}

\author{
by ADRIAN M. RILEY, RODNEY Y. SHARP and HOSSEIN ZAKERI
}

(Received 21 September, 1983)

Introduction. Let $A$ be a commutative Noetherian ring (with non-zero identity). The Cousin complex $C(A)$ for $A$ is described in $[6,82]$ : it is a complex of $A$-modules and A-homomorphisms

$$
0 \stackrel{d^{-2}}{\longrightarrow} A \stackrel{d^{-1}}{\longrightarrow} A^{0} \stackrel{d^{0}}{\longrightarrow} A^{1} \longrightarrow \ldots \longrightarrow A^{n} \stackrel{d^{n}}{\longrightarrow} A^{n+1} \longrightarrow \ldots
$$

with the property that, for each $n \geqslant 0$,

$$
A^{n}=\bigoplus_{\substack{p \in S p e c(A) \\ \text { ht } p=n}}^{\bigoplus}\left(\text { coker } d^{n-2}\right)_{p}
$$

Cohen-Macaulay rings may be characterized in terms of the Cousin complex: $A$ is a Cohen-Macaulay ring if and only if $C(A)$ is exact $[6,(4.7)]$. Also the Cousin complex provides a natural minimal injective resolution for a Gorenstein ring: see [6, (5.4)].

Various more general Cousin complexes can be constructed. If $M$ is an $A$-module, then we can construct the Cousin complex $C(M)$ for $M$ as in $[6, \S 2]$ : for that we concentrate attention on the prime ideals in $\operatorname{Supp}(M)$, the support of $M$. Again, for $M$ non-zero and finitely generated, we have that $M$ is a Cohen-Macaulay $A$-module if and only if $C(M)$ is exact: see [7, (2.4)]. More generally still, we can, for any filtration $\mathscr{F}$ [11, $1.1]$ of $\operatorname{Spec}(A)$ that admits $M$, construct the Cousin complex $C(\mathscr{F}, M)$ for $M$ with respect to $\mathscr{F}[11,1.3]$. If we use for $\mathscr{F}$ the $M$-height filtration $\mathscr{H}(M)$ of $\operatorname{Spec}(A)[11,1.2]$, then $C(\mathscr{H}(M), M)$ is just the Cousin complex $C(M)$ mentioned earlier. When $A$ is local, use of the dimension filtration $\mathscr{D}(A)[11,1.2]$ of $\operatorname{Spec}(A)$ permits a characterization of balanced big Cohen-Macaulay $A$-modules (a (not necessarily finitely generated) $A$-module $X$ is a balanced big Cohen-Macaulay A-module [10, p. 229] if every system of parameters for $A$ is an $X$-sequence). It turns out that $M$ is a balanced big Cohen-Macaulay $A$-module if and only if $C(\mathscr{D}(A), M)$ is exact and $M \neq m M$ (where $m$ denotes the maximal ideal of $A$ ).

Although Cousin complexes do provide satisfactory characterizations of various Cohen-Macaulay properties, they have the disadvantage that their construction is rather complicated, and this perhaps makes them difficult to work with. The purpose of this paper is to show that every Cousin complex for an $A$-module $M$ which has only finitely many minimal associated prime ideals may be described, up to isomorphism, as a complex of modules of generalized fractions (such modules were introduced in [13]); this description is perhaps simpler and easier to work with.

Given a filtration $\mathscr{F}$ of $\operatorname{Spec}(A)$ that admits such an $M$ we shall show that there is induced a chain of triangular subsets on $A$. Such a chain is a family $U=\left(U_{i}\right)_{i \in \mathbb{N}}$ (where $\mathbb{N}$

Glasgow Math. J. 26 (1985) 51-67. 
denotes the set of positive integers) for which the following conditions are satisfied:

(i) $U_{i}$ is a triangular subset $[13,2.1]$ of $A^{i}$ for all $i \in \mathbb{N}$;

(ii) (1) $\in U_{1}$;

(iii) whenever $\left(u_{1}, \ldots, u_{i}\right) \in U_{i}$ with $i \in \mathbb{N}$, then $\left(u_{1}, \ldots, u_{i}, 1\right) \in U_{i+1}$;

(iv) whenever $\left(u_{1}, \ldots, u_{i}\right) \in U_{i}$ with $1<i \in \mathbb{N}$, then $\left(u_{1}, \ldots, u_{i-1}\right) \in U_{i-1}$.

Each $U_{i}$ leads to a module of generalized fractions $U_{i}^{-i} M[13]$, and we can, in fact, arrange these modules into a complex

$$
0 \rightarrow M \stackrel{e^{0}}{\rightarrow} U_{1}^{-1} M \stackrel{e^{1}}{\rightarrow} U_{2}^{-2} M \rightarrow \ldots \rightarrow U_{n}^{-n} M \stackrel{e^{n}}{\rightarrow} U_{n+1}^{-n-1} M \rightarrow \ldots
$$

denoted by $C(\mathcal{U}, M)$, for which $e^{0}(m)=\frac{m}{(1)}$ for all $m \in M$ and

$$
e^{i}\left(\frac{x}{\left(u_{1}, \ldots, u_{i}\right)}\right)=\frac{x}{\left(u_{1}, \ldots, u_{i}, 1\right)}
$$

for all $i \in \mathbb{N}, x \in M$ and $\left(u_{1}, \ldots, u_{i}\right) \in U_{i}$ : this is easy to verify $([13,3.3(i i)]$ is helpful). In the situation that concerns us here, we shall show that this complex $C(\mathcal{U}, M)$ is, in fact, isomorphic to the Cousin complex $C(\mathscr{F}, M)$. The exact definition of the chain $\mathcal{U}$ will be given in $\S 2$; however, in the special case in which $M=A$ and $\mathscr{F}$ is the height filtration [11, 1.2] of $\operatorname{Spec}(A)$, it turns out that, for all $i \in \mathbb{N}$,

$$
U_{i}=\left\{\left(u_{1}, \ldots, u_{i}\right) \in A^{i}: \operatorname{ht}\left(A u_{1}+\ldots+A u_{i}\right) \geqslant j \text { for all } j=1, \ldots, i\right\} .
$$

(We interpret ht $A$ as $\infty$.)

The existence of the above-mentioned isomorphism will be established in $\S 3$. Impetus for the present work came from two results in Zakeri's $\mathrm{Ph} . \mathrm{D}$. thesis [15]: he established results of a similar type in the special cases where (a) $M$ is a non-zero finitely generated Cohen-Macaulay $A$-module and $\mathscr{F}=\mathscr{H}(M)[15$, Chapter III, Theorem (3.7)] and (b) $A$ is local, $M$ is a balanced big Cohen-Macaulay $A$-module and $\mathscr{F}=\mathscr{D}(A)[15$, Chapter IV, Theorem (3.15)]. In both these situations the Cousin complex concerned is exact, and, indeed, Zakeri's arguments depended on the results on the structure of certain exact Cousin complexes in [12]. These results are phrased in terms of the concept of repeated division in Cousin complexes. In fact, in $\$ 3$ below we shall use repeated division to provide a simple description of our main isomorphism.

This will enable us to show that special cases of the isomorphism lead to Zakeri's two results cited above, and to extend one of the results on the structure of Cousin complexes from [12] to more general, non-exact situations.

We believe that the results of this paper provide additional evidence in support of the view that the concept of module of generalized fractions is worth further study.

1. Notation and preliminary results about Cousin complexes. Throughout the paper, $A$ will denote a commutative Noetherian ring (with non-zero identity) and $M$ will denote an $A$-module. We shall use the terminology of $[11, \S 1]$ concerning the Cousin complex for $M$ with respect to a filtration of $\operatorname{Spec}(A)$ that admits $M$; in particular, 
$\mathscr{F}=\left(F_{i}\right)_{i \geq 0}$ will always denote a filtration of $\operatorname{Spec}(A)$ that admits $M$, and

$$
0 \stackrel{d^{-2}}{\longrightarrow} M \stackrel{d^{-1}}{\longrightarrow} M^{0} \stackrel{d^{0}}{\longrightarrow} M^{1} \longrightarrow \ldots \longrightarrow M^{i} \stackrel{d^{\prime}}{\longrightarrow} M^{i+1} \longrightarrow \ldots
$$

will always denote the Cousin complex $C(\mathscr{F}, M)$ for $M$ with respect to $\mathscr{F}$. We use $\mathbb{N}$ (respectively $\mathbb{N}_{0}$ ) to denote the set of positive (respectively non-negative) integers. Two facts from [11, §1] will be crucial in the argument below, and are stated here for convenience.

(1.1) Proposmion [11, 1.4(i)]. (i) For each $n \in \mathbb{N}_{0}$,

$$
\operatorname{Supp}\left(M^{n}\right) \subseteq \operatorname{Supp}\left(\operatorname{coker} d^{n-2}\right) \subseteq F_{n} .
$$

(ii) For each $n \in \mathbb{N}_{0}$, Supp $\left(\operatorname{ker} d^{n-1} / \mathrm{im} d^{n-2}\right) \subseteq F_{n+1}$.

The proof of the following uses basic ideas concerning the Cousin complex.

(1.2) Lemma. Let $n \in \mathbb{N}_{0}$.

(i) For each $\mathfrak{p} \in \partial F_{n}$, there is an A-isomorphism

$$
\eta(\mathfrak{p}):\left(M^{n}\right)_{\mathfrak{p}} \rightarrow\left(\text { coker } d^{n-2}\right)_{\mathfrak{p}}
$$

such that, for $\beta \in M^{n}$ with component $\beta_{\mathfrak{p}} / s_{\mathrm{p}}$ in the summand (coker $d^{n-2}$ ) (where $\beta_{\mathfrak{p}} \in$ coker $d^{n-2}$ and $s_{\mathfrak{p}} \in A \backslash \mathfrak{p}$ ) and $t \in A \backslash \mathfrak{p}$, we have

$$
\eta(\mathfrak{p})\left(\frac{\beta}{t}\right)=\frac{\beta_{\mathfrak{p}}}{t s_{\mathfrak{p}}} .
$$

(ii) Since $\operatorname{Supp}\left(M^{n}\right) \subseteq F_{n}$ (by (1.1)(i)) and $\partial F_{n}$ is low with respect to $F_{n}$, it follows from [6, (2.2) and (2.3)] that there is an A-homomorphism

$$
\omega^{n}: M^{n} \rightarrow \underset{p \in \partial F_{n}}{\oplus}\left(M^{n}\right)_{p}
$$

such that, for $\beta \in M^{n}$ and $\mathfrak{p} \in \partial F_{n}$, the component of $\omega^{n}(\beta)$ in $\left(M^{n}\right)_{\mathfrak{p}}$ is $\beta / 1$. In fact, $\omega^{n}=\left[\underset{p \in \partial F_{n}}{\oplus} \eta(\mathfrak{p})\right]^{-1}$, and so is an isomorphism.

Proof. (i) This follows from [6, (2.5)(ii)].

(ii) It is straightforward to check that

is the identity mapping.

$$
\left[\underset{p \in \partial F_{n}}{\oplus} \eta(p)\right] \circ \omega^{n}: M^{n} \rightarrow M^{n}
$$

We shall need the concept of repeated division in Cousin complexes, introduced in $[12, \S 2]$. We recall the main ingredients.

(1.3) LEMma [12, (2.1)]. Let $U, U^{\prime}$ be subsets of $\operatorname{Spec}(A)$ such that $U^{\prime} \subseteq U$ and $U \backslash U^{\prime}$ is low with respect to $U$. Let $X$ be an A-module with $\operatorname{Supp}(X) \subseteq U$ and put 
$H=\underset{\mathfrak{p} \in U \backslash U^{\prime}}{\oplus} X_{\mathfrak{p}}$. Suppose that $x \in X$ and $a \in A$ are such that

$$
\operatorname{ann}(x)+A a \nsubseteq \mathrm{q}
$$

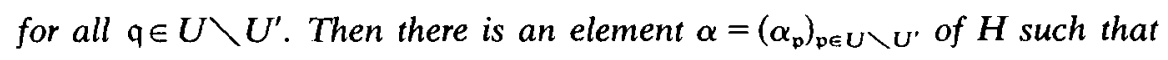

We denote $\alpha$ by $x \div a$.

$$
\alpha_{\mathfrak{p}}=\left\{\begin{array}{l}
0 \text { for } \mathfrak{p} \in U \backslash U^{\prime} \text { with } \operatorname{ann}(x) \nsubseteq \mathfrak{p}, \\
\frac{x}{a} \text { for } \mathfrak{p} \in U \backslash U^{\prime} \text { with } \operatorname{ann}(x) \subseteq \mathfrak{p} .
\end{array}\right.
$$

This idea is extended to Cousin complexes as follows.

(1.4) Definition. For each $i \in \mathbb{N}_{0}$, let $\pi^{i}: M^{i} \rightarrow$ coker $d^{i-1}$ denote the canonical epimorphism.

Let $x \in M$ and let $a_{1}, a_{2}, \ldots, a_{t} \in A$. We say that $x$ is divisible by the sequence $a_{1}$, $a_{2}, \ldots, a_{1}$, or that $a_{1}, a_{2}, \ldots, a_{1}$ divides $x$, with respect to $\mathscr{F}$, if the following conditions are satisfied:

(1) $\operatorname{ann}(x)+A a_{1} \nsubseteq \mathfrak{p}$ for each $\mathfrak{p} \in \partial F_{0}$, so that the element $x \div a_{1}$ of $M^{0}$ may be constructed;

(2) $\operatorname{ann}\left(\pi^{0}\left(x \div a_{1}\right)\right)+A a_{2} \nsubseteq \mathfrak{p}$ for each $\mathfrak{p} \in \partial F_{1}$, so that, by (1.3), the element $\pi^{0}\left(x \div a_{1}\right) \div a_{2}$ of $M^{1}$ may be formed; this element is denoted by $x \div a_{1}, a_{2}$;

$$
\operatorname{ann}\left(\pi^{t-2}\left(x \div a_{1}, a_{2}, \ldots, a_{t-1}\right)\right)+A a_{t} \nsubseteq \mathfrak{p} \text { for each } \mathfrak{p} \in \partial F_{t-1} \text {, }
$$

so that the element $\pi^{t-2}\left(x \div a_{1}, a_{2}, \ldots, a_{t-1}\right) \div a_{t}$ of $M^{t-1}$ may be formed; this element is denoted by $x \div a_{1}, a_{2}, \ldots, a_{1}$ and is referred to as the result of dividing $x$ by the sequence $a_{1}, a_{2}, \ldots, a_{1}$.

Terminology and notation concerning triangular subsets of $A^{n}$ (for $n \in \mathbb{N}$ ) and modules of generalized fractions will be the same as that used in [13, §2]. In particular, $D_{n}(A)$ denotes the set of $n \times n$ lower triangular matrices over $A$. We include here for the reader's convenience one basic lemma about generalized fractions.

(1.5) LeMMA. Let $U$ be a triangular subset of $A^{n}$, and suppose that $m \in M$ and $\left(u_{1}, \ldots, u_{n}\right) \in U$ are such that $\frac{u_{n} m}{\left(u_{1}, \ldots, u_{n}\right)}=0$ in $U^{-n} M$. Then $\frac{m}{\left(u_{1}, \ldots, u_{n}\right)}=0$.

Proof. There exist $\left(w_{1}, \ldots, w_{n}\right) \in U$ and $\mathbf{H}=\left[h_{i j}\right] \in D_{n}(A)$ such that

$$
\mathbf{H}\left[u_{1} \ldots u_{n}\right]^{T}=\left[w_{1} \ldots w_{n}\right]^{T} \quad \text { and } \quad|\mathbf{H}| u_{n} m \in\left(\sum_{i=1}^{n-1} A w_{i}\right) M
$$

Hence

$$
h_{11} \ldots h_{n-1 n-1}\left(w_{n}-\sum_{i=1}^{n-1} h_{n i} u_{i}\right) m \in\left(\sum_{i=1}^{n-1} A w_{i}\right) M
$$


Therefore, by $[13,2.2], h_{11} \ldots h_{n-1 n-1} w_{n} m \in\left(\sum_{i=1}^{n-1} A w_{i}\right) M$; hence, by $[13,3.3(i i)]$,

$$
\frac{h_{11} \ldots h_{n-1 n-1} w_{n} m}{\left(w_{1}, \ldots, w_{n-1}, w_{n}^{2}\right)}=0
$$

in $U^{-n} M$. It follows from this that

$$
\frac{h_{11} \ldots h_{n-1 n-1} h_{n n} m}{\left(w_{1}, \ldots, w_{n-1}, w_{n}\right)}=0
$$

and the desired conclusion is a consequence of this because

$$
\mathbf{H}\left[u_{1} \ldots u_{n}\right]^{\mathrm{T}}=\left[w_{1} \ldots w_{n}\right]^{\mathrm{T}} \text {. }
$$

2. The induced chain of triangular subsets. In this section we shall assume throughout that $\operatorname{Ass}(M)$ contains only finitely many minimal members and we shall show that, under this assumption, the filtration $\mathscr{F}$ that admits $M$ induces a chain of triangular subsets on $A$. We shall also establish some properties of the resulting complex of modules of generalized fractions.

For each $n \in \mathbb{N}$, define

$$
U_{n}=\left\{\left(u_{1}, \ldots, u_{n}\right) \in A^{n}: \text { for each } i=1, \ldots, n, \sum_{j=1}^{i} A u_{i} \nsubseteq p \text { for all } \mathfrak{p} \in \partial F_{i-1} \cap \operatorname{Supp}(M)\right\},
$$

and let $\mathcal{U}$ denote the family $\left(U_{i}\right)_{i \gg 1}$. Our first aim is to show that $\mathcal{U}$ is a chain of triangular subsets on $A$.

(2.1) Lemma. (Ass $(M)$ contains only finitely many minimal members.) Let $n \in \mathbb{N}$ and $\left(u_{1}, \ldots, u_{n}\right) \in U_{n}$. Let $\mathfrak{p} \in \partial F_{n} \cap \operatorname{Supp}(M)$ with $\sum_{i=1}^{n} A u_{i} \subseteq \mathfrak{p}$. Let $\operatorname{Min}(M)$ denote the set of minimal associated prime ideals of $M$. Then $\mathfrak{p}$ is a minimal prime ideal of the ideal

$$
\mathfrak{a}=\sum_{i=1}^{n} A u_{i}+\bigcap_{\mathfrak{q} \in \operatorname{Min}(M)} \mathfrak{q} \text {. }
$$

In particular, there are only finitely many primes in $\partial F_{n} \cap \operatorname{Supp}(M)$ that contain $\sum_{i=1}^{n} A u_{i}$.

Also $\partial F_{0} \cap \operatorname{Supp}(M) \subseteq \operatorname{Min}(M)$, and so is finite.

Proof. Suppose that there exists $\mathfrak{p}^{\prime} \in \operatorname{Spec}(A)$ with $a \subseteq \mathfrak{p}^{\prime} \subseteq \mathfrak{p}$. Then $\mathfrak{p}^{\prime}$ contains a member of $\operatorname{Min}(M)$, and so $\mathfrak{p}^{\prime} \in \operatorname{Supp}(M) \subseteq F_{0}$. Also, $\sum_{i=1}^{n} A u_{i} \subseteq \mathfrak{p}^{\prime}$, so that $\mathfrak{p}^{\prime} \notin \partial F_{i}$ for each $i=0, \ldots, n-1$. Thus $\mathfrak{p}^{\prime} \in F_{n}$. Since $\mathfrak{p}$ is a minimal member of $F_{n}$, we see that $\mathfrak{p}=\mathfrak{p}^{\prime}$.

The final claim is an easy consequence of the hypotheses.

(2.2) Remark. Note that the conclusion of (2.1) need not hold in the case of a module which has infinitely many minimal associated prime ideals. For suppose that $\boldsymbol{A}$ is 
a regular local ring of dimension 3 with maximal ideal $m=A a_{1}+A a_{2}+A a_{3}$ and put

$$
X=\bigoplus_{\substack{\mathfrak{p} \in \operatorname{Spec}(A) \backslash\left\{A a_{1}\right\} \\ \text { ht } \mathfrak{p}=1}} A / p .
$$

Then $\operatorname{Supp}(X)=\left\{\mathfrak{p} \in \operatorname{Spec}(A):\right.$ ht $p \geqslant 1$ and $\left.\mathfrak{p} \neq A a_{1}\right\}$. Thus if $\mathscr{G}=\left(G_{i}\right)_{i \geqslant 0}$ is the filtration of $\operatorname{Spec}(A)$ given by $G_{i}=\{\mathfrak{p} \in \operatorname{Spec}(A):$ ht $p \geqslant i+1\}$ for each $i \geqslant 0$, then $\mathscr{G}$ admits $X$. Now $a_{1} \notin \mathfrak{p}$ for each $\mathfrak{p} \in \partial G_{0} \cap \operatorname{Supp}(X)$, but there are infinitely many prime ideals in $\partial G_{1} \cap \operatorname{Supp}(X)$ which contain $A a_{1}$, because there are infinitely many primes of height 2 that contain $A a_{1}$.

The concept of a chain of triangular subsets on $A$ was defined in the introduction.

(2.3) Proposition. (Ass $(M)$ contains only finitely many minimal members.) $u=$ $\left(U_{i}\right)_{i \geqslant 1}$ is a chain of triangular subsets on $A$.

Proof. We show that $U_{n}$ is a triangular subset of $A^{n}$ for each $n \in \mathbb{N}$.

Now $U_{1}$ is a multiplicatively closed and hence $[13,3.1]$ triangular subset of $A^{1}$. Assume, inductively, that $n>1$ and $U_{n-1}$ is known to be a triangular subset of $A^{n-1}$. Clearly $U_{n} \neq \varnothing$ and, whenever $\left(u_{1}, \ldots, u_{n}\right) \in U_{n}$ and $\alpha_{1}, \ldots, \alpha_{n} \in \mathbb{N}$, then $\left(u_{1}^{\alpha_{1}}, \ldots, u_{n}^{\alpha_{n}}\right) \in$ $U_{n}$ also. Let $\left(u_{1}, \ldots, u_{n}\right),\left(v_{1}, \ldots, v_{n}\right) \in U_{n}$. By the inductive hypothesis, there exists $\left(w_{1}, \ldots, w_{n-1}\right) \in U_{n-1}$ such that, for all $i=1, \ldots, n-1$,

$$
w_{i} \in\left(A u_{1}+\ldots+A u_{i}\right) \cap\left(A v_{1}+\ldots+A v_{i}\right) \text {. }
$$

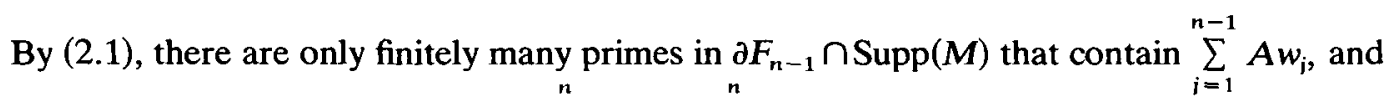
none of these primes can contain $\sum_{j=1}^{n} A u_{j}$ or $\sum_{j=1}^{n} A v_{j}$. Hence there exists

$$
w_{n} \in\left(A u_{1}+\ldots+A u_{n}\right) \cap\left(A v_{1}+\ldots+A v_{n}\right)
$$

such that $w_{n} \notin \mathfrak{p}$ for each $\mathfrak{p}$ for which $\sum_{i=1}^{n-1} A w_{i} \subseteq \mathfrak{p} \in \partial F_{n-1} \cap \operatorname{Supp}(M)$. It follows that $\left(w_{1}, \ldots, w_{n}\right) \in U_{n}$.

Hence $U_{n}$ is a triangular subset of $A^{n}$, and the inductive step is complete. All the remaining claims are clear.

(2.4) Notation. (Ass $(M)$ contains only finitely many minimal members.) In view of (2.3), we may form, in the manner indicated in the introduction, the complex $C(\mathcal{U}, M)$ : let this be denoted by

$$
0 \stackrel{e^{-1}}{\longrightarrow} M \stackrel{e^{0}}{\longrightarrow} U_{1}^{-1} M \stackrel{e^{1}}{\longrightarrow} \ldots \longrightarrow U_{n}^{-n} M \stackrel{e^{n}}{\longrightarrow} U_{n+1}^{-n-1} M \longrightarrow \ldots
$$

It is convenient to make the convention whereby $U_{0}^{-0} M$ is interpreted as $M$. For each $n \in \mathbb{N}_{0}$, we shall use $\theta^{n}: U_{n}^{-n} M \rightarrow \operatorname{coker} e^{n-1}$ to denote the canonical epimorphism.

Our next step is to show that $C(\mathscr{U}, M)$ has properties analogous to those of $C(\mathscr{F}, M)$ described in (1.1) and (1.2)(ii). 
(2.5) THEOREM. (Ass(M) contains only finitely many minimal members.)

(i) For each $n \in \mathbb{N}, \quad \operatorname{Ass}\left(U_{n}^{-n} M\right) \subseteq \partial F_{n-1} \cap \operatorname{Supp}(M) \quad$ and $\operatorname{Supp}\left(U_{n}^{-n} M\right) \subseteq$ $F_{n-1} \cap \operatorname{Supp}(M)$.

(ii) For each $n \in \mathbb{N}_{0}$, Supp(coker $\left.e^{n-1}\right) \subseteq F_{n} \cap \operatorname{Supp}(M)$.

(iii) For each $n \in \mathbb{N}_{0}$,

$$
\operatorname{ker} e^{n} / \operatorname{im} e^{n-1}=\left\{x \in \operatorname{coker} e^{n-1}: \operatorname{Supp}(A x) \subseteq F_{n+1} \cap \operatorname{Supp}(M)\right\},
$$

so that $\operatorname{Supp}\left(\operatorname{ker} e^{n} / \operatorname{im} e^{n-1}\right) \subseteq F_{n+1} \cap \operatorname{Supp}(M)$.

Proof. (i) Let $n \in \mathbb{N}$ and $\mathfrak{p} \in \operatorname{Ass}\left(U_{n}^{-n} M\right)$. Thus there exist $m \in M$ and $\left(u_{1}, \ldots, u_{n}\right) \in U_{n}$ such that $\mathfrak{p}=\operatorname{ann}\left(\frac{m}{\left(u_{1}, \ldots, u_{n}\right)}\right)$. Hence, by $[13,(3.3)($ ii $)]$, ann $(m)+\sum_{j=1}^{n-1} A u_{i} \subseteq p$. Thus $\mathfrak{p} \in \operatorname{Supp}(M) \subseteq F_{0}$, and $\sum_{i=1}^{n-1} A u_{i} \subseteq \mathfrak{p}$. Thus, if $n>1$, we have $\mathfrak{p} \notin \partial F_{i-1}$ for each $i=$ $1, \ldots, n-1$. Therefore, in any event, $\mathfrak{p} \in F_{n-1} \cap \operatorname{Supp}(M)$.

Now suppose that $\mathfrak{p} \notin \partial F_{n-1}$. By (2.1), there are only finitely many primes in $\partial F_{n-1} \cap \operatorname{Supp}(M)$ which contain $\sum_{j=1}^{n-1} A u_{j}$ : let these be $q_{1}, \ldots, q_{r}$. Then there exists $u^{\prime} \in$ $\mathfrak{p} \backslash \bigcup_{j=1}^{r} \mathfrak{q}_{j}$, so that $u^{\prime} u_{n} \in \mathfrak{p} \backslash \bigcup_{j=1}^{r} q_{j}$. It follows that $\left(u_{1}, \ldots, u_{n-1}, u^{\prime} u_{n}\right) \in U_{n}$. We have

$$
\frac{\left(u^{\prime} u_{n}\right) u^{\prime} m}{\left(u_{1}, \ldots, u_{n-1}, u^{\prime} u_{n}\right)}=\frac{\left(u^{\prime} u_{n}\right) m}{\left(u_{1}, \ldots, u_{n-1}, u_{n}\right)}=0
$$

since $u^{\prime} u_{n} \in \mathfrak{p}$. Hence, by 1.5 ,

$$
\frac{m}{\left(u_{1}, \ldots, u_{n-1}, u_{n}\right)}=\frac{u^{\prime} m}{\left(u_{1}, \ldots, u_{n-1}, u^{\prime} u_{n}\right)}=0 .
$$

This contradiction shows that $\mathfrak{p} \in \partial F_{n-1} \cap \operatorname{Supp}(M)$.

Hence $\operatorname{Ass}\left(U_{n}^{-n} M\right) \subseteq \partial F_{n-1} \cap \operatorname{Supp}(M)$. Let $q \in \operatorname{Supp}\left(U_{n}^{-n} M\right)$, so that $\mathfrak{q} \supseteq \mathfrak{p}$ for some $\mathfrak{p} \in \operatorname{Ass}\left(U_{n}^{-n} M\right)$. Since $p \in F_{n-1}$, it follows that $q \notin \partial F_{i}$ for each $i=0,1, \ldots, n-2$. But $\mathfrak{q} \in \operatorname{Supp}(M) \subseteq F_{0}$, and so $q \in F_{n-1} \cap \operatorname{Supp}(M)$.

(ii) Let $n \in \mathbb{N}$, and let $\alpha=\frac{m}{\left(u_{1}, \ldots, u_{n}\right)} \in U_{n}^{-n} M$ (where $m \in M$ and $\left(u_{1}, \ldots, u_{n}\right) \in U_{n}$ ).
By $[13,3.3]$,

$$
\operatorname{ann}(m)+A u_{1}+\ldots+A u_{n} \subseteq \operatorname{ann}\left(\theta^{n}(\alpha)\right) \text {. }
$$

It follows that $\operatorname{Supp}\left(\operatorname{coker} e^{n-1}\right) \subseteq F_{n} \cap \operatorname{Supp}(M)$.

(iii) First of all, by $[13,3.1]$,

$$
\begin{aligned}
\operatorname{ker} e^{0} & =\left\{x \in M: u_{1} x=0 \text { for some }\left(u_{1}\right) \in U_{1}\right\} \\
& =\left\{x \in M: \operatorname{Supp}(A x) \subseteq F_{1} \cap \operatorname{Supp}(M)\right\}
\end{aligned}
$$

since, by (2.1), $\partial F_{0} \cap \operatorname{Supp}(M)$ is finite.

For the remainder of the proof suppose that $n \in \mathbb{N}$. Let $m \in M,\left(u_{1}, \ldots, u_{n}\right) \in U_{n}$ be 
such that $y=\frac{m}{\left(u_{1}, \ldots, u_{n}\right)} \in \operatorname{ker} e^{n}$. Set $x=\theta^{n}(y)$. Now there exist $\mathbf{H}=\left[h_{i j}\right] \in D_{n+1}(\mathrm{~A})$ and $\left(v_{1}, \ldots, v_{n}, v_{n+1}\right) \in U_{n+1}$ such that

$$
\mathbf{H}\left[u_{1} \ldots u_{n} 1\right]^{\mathrm{T}}=\left[v_{1} \ldots v_{n} v_{n+1}\right]^{\mathrm{T}}
$$

and

$$
|\mathbf{H}| m \in\left(\sum_{i=1}^{n} A v_{i}\right) M
$$

Now, by $[13,3.3], \sum_{i=1}^{n} A v_{i} \subseteq \sum_{i=1}^{n} A u_{i} \subseteq \operatorname{ann}(x)$. Also, by (1) and (2),

$$
\frac{h_{n+1 n+1} m}{\left(u_{1}, \ldots, u_{n}\right)}=\frac{|\mathbf{H}| m}{\left(v_{1}, \ldots, v_{n}\right)}=\frac{m^{\prime}}{\left(v_{1}, \ldots, v_{n-1}, 1\right)}
$$

for some $m^{\prime} \in M$. Thus $h_{n+1}+1 \in \operatorname{ann}(x)$; hence, by (1), $v_{n+1} \in \operatorname{ann}(x)$. Thus $\sum_{i=1}^{n+1} A v_{i} \subseteq$ $\operatorname{ann}(x)$. Thus $\operatorname{ann}(x) \nsubseteq \mathfrak{p}$ for each $\mathfrak{p} \in \partial F_{i}$ for each $i=0, \ldots, n$. Since $\operatorname{ann}(m) \subseteq \operatorname{ann}(x)$, it follows that $\operatorname{Supp}(A x) \subseteq F_{n+1} \cap \operatorname{Supp}(M)$.

Conversely, let $x \in \operatorname{coker} e^{n-1}$ be such that $\operatorname{Supp}(A x) \subseteq F_{n+1} \cap \operatorname{Supp}(M)$. Thus $x=$ $\theta^{n}\left(\frac{m}{\left(u_{1}, \ldots, u_{n}\right)}\right)$ for some $m \in M,\left(u_{1}, \ldots, u_{n}\right) \in U_{n}$. By $(2.1)$, there exists $u_{n+1} \in \operatorname{ann}(x)$ such that $\left(u_{1}, \ldots, u_{n}, u_{n+1}\right) \in U_{n+1}$. Hence there exist $m^{\prime} \in M,\left(v_{1}, \ldots, v_{n-1}, 1\right)$, $\left(w_{1}, \ldots, w_{n}\right) \in U_{n}$ and $\mathbf{H}=\left[h_{i j}\right], \mathbf{K}=\left[k_{i j}\right] \in D_{n}(A)$ such that

$$
\mathbf{H}\left[u_{1} \ldots u_{n}\right]^{T}=\left[w_{1} \ldots w_{n}\right]^{T}=\mathbf{K}\left[v_{1} \ldots v_{n-1} 1\right]^{T}
$$

and

$$
\text { |H }\left|u_{n+1} m-\right| \mathbf{K} \mid m^{\prime} \in\left(\sum_{i=1}^{n-1} A w_{i}\right) M \text {. }
$$

By (3) and (2.1), there exists $w_{n+1} \in \sum_{i=1}^{n+1} A u_{i}$ such that $\left(w_{1}, \ldots, w_{n}, w_{n+1}\right) \in U_{n+1}$; we have $w_{n+1}=\sum_{i=1}^{n+1} h_{n+1 i} u_{i}$ with $h_{n+1 i} \in A($ for $i=1, \ldots, n+1)$. Set

$$
\mathbf{H}^{\prime}=\left[\begin{array}{c|c}
\mathbf{H} & \mathbf{0} \\
\hline h_{n+11} \ldots h_{n+1 n} & h_{n+1 n+1}
\end{array}\right],
$$

so that $\mathbf{H}^{\prime} \in D_{n+1}(A)$ and $\mathbf{H}^{\prime}\left[u_{1} \ldots u_{n} u_{n+1}\right]^{\mathrm{T}}=\left[w_{1} \ldots w_{n} w_{n+1}\right]^{\mathrm{T}}$. We have

$$
\left|\mathbf{H}^{\prime}\right| u_{n+1} m=h_{n+1 n+1}\left(|\mathbf{H}| u_{n+1} m-|\mathbf{K}| m^{\prime}\right)+h_{n+1 n+1}|\mathbf{K}| m^{\prime} \in\left(\sum_{i=1}^{n} A w_{i}\right) M
$$

by (3), (4) and $[13,2.2]$. Thus

$$
e^{n}\left(\frac{m}{\left(u_{1}, \ldots, u_{n}\right)}\right)=\frac{m}{\left(u_{1}, \ldots, u_{n}, 1\right)}=\frac{u_{n+1} m}{\left(u_{1}, \ldots, u_{n}, u_{n+1}\right)}=0 .
$$

Hence $x \in \operatorname{ker} e^{n} / \mathrm{im} e^{n-1}$, as required. 
(2.6) Theorem. (Ass $(M)$ contains only finitely many minimal members.) Let $n \in \mathbb{N}$. Since $\operatorname{Supp}\left(U_{n}^{-n} M\right) \subseteq F_{n-1}$ (by (2.5)), it follows from [6, (2.2) and (2.3)] that there is an A-homomorphism

$$
\gamma^{n}: U_{n}^{-n} M \rightarrow \underset{p \in \partial F_{n-1}}{\bigoplus}\left(U_{n}^{-n} M\right)_{\mathfrak{p}}
$$

such that, for $\alpha \in U_{n}^{-n} M$ and $\mathfrak{p} \in \partial F_{n-1}$, the component of $\gamma^{n}(\alpha)$ in the summand $\left(U_{n}^{-n} M\right)_{p}$ is $\alpha / 1$.

The homomorphism $\gamma^{n}$ is an isomorphism.

Proof. By (2.5)(i), Ass(Ker $\left.\gamma^{n}\right) \subseteq \partial F_{n-1}$, and so $\gamma^{n}$ is injective.

Let $\mathfrak{p}_{1} \in \partial F_{n-1}, m \in M,\left(u_{1}, \ldots, u_{n}\right) \in U_{n}, s \in A \backslash \mathfrak{p}_{1}$, and let $\delta$ denote the element of $\underset{p \in \partial F_{n-1}}{\bigoplus}\left(U_{n}^{-n} M\right)_{p}$ having component

$$
\delta_{p_{1}}=\frac{\frac{m}{\left(u_{1}, \ldots, u_{n}\right)}}{s}
$$

in $\left(U_{n}^{-n} M\right)_{p_{1}}$ and zero components in all the other summands. It is enough to show that $\delta \in \operatorname{im} \gamma^{\prime \prime}$, and we can assume that $\delta_{p_{1}} \neq 0$.

Let $x=\frac{m}{\left(u_{1}, \ldots, u_{n}\right)}$ and $a=\operatorname{ann}(x)$. By $(2.5)(\mathrm{i})$, every associated prime of $a$ is in $\partial F_{n-1} \cap \operatorname{Supp}(M)$; also, since $a \subseteq \mathfrak{p}_{1}$ and $\mathfrak{p}_{1} \in \partial F_{n-1}$, it now follows that $\mathfrak{p}_{1}$ is itself an associated prime of $\mathbf{a}$.

Let the associated primes of $a$ be $\mathfrak{p}_{1}, \ldots, \mathfrak{p}_{k}(k \geqslant 1)$ and let

$$
\mathfrak{a}=\mathfrak{q}_{1} \cap \ldots \cap \mathfrak{q}_{k}
$$

be a minimal primary decomposition of $\mathfrak{a}$, where $r\left(\mathfrak{q}_{i}\right)=\mathfrak{p}_{i} \quad(i=1, \ldots, k)$. Since $\operatorname{ann}(m)+\sum_{i=1}^{n-1} A u_{i} \subseteq \operatorname{ann}(x) \subseteq \mathfrak{p}_{1}$, it follows that $u_{n} \notin \mathfrak{p}_{1}$. Also, in $U_{n}^{-n} M$,

hence, in $\left(U_{n}^{-n} M\right)_{\mathfrak{p}_{1}}$,

$$
s u_{n}\left(\frac{m}{\left(u_{1}, \ldots, u_{n}\right)}\right)=s\left(\frac{m}{\left(u_{1}, \ldots, u_{n-1}, 1\right)}\right)
$$

$$
\delta_{\mathfrak{p}_{1}}=\frac{\frac{m}{\left(u_{1}, \ldots, u_{n}\right)}}{s}=\frac{m}{\frac{\left(u_{1}, \ldots, u_{n-1}, 1\right)}{s u_{n}}}
$$

Now there exists $t \in \bigcap_{i=2}^{k} \mathfrak{q}_{i} \backslash \mathfrak{p}_{1}$, and, in $\left(U_{n}^{-n} M\right)_{\mathfrak{p}_{1}}$, we have

$$
\delta_{p_{1}}=\frac{m}{\frac{\left(u_{1}, \ldots, u_{n-1}, 1\right)}{s u_{n}}}=\frac{t m}{\frac{\left(u_{1}, \ldots, u_{n-1}, 1\right)}{t s u_{n}}} .
$$


Also, it follows from (1.5) that

$$
\operatorname{ann}\left(\frac{t m}{\left(u_{1}, \ldots, u_{n-1}, 1\right)}\right)=\operatorname{ann}(t x)=(a: t)=\bigcap_{i=1}^{k}\left(\mathfrak{q}_{i}: t\right)=\mathfrak{q}_{1} .
$$

Next we note that, if $Q$ denotes $\left\{\mathfrak{p} \in \partial F_{n-1} \cap \operatorname{Supp}(M): \sum_{i=1}^{n-1} A u_{i} \subseteq \mathfrak{p}\right\}$, then $A t s u_{n}+\mathfrak{q}_{1} \nsubseteq \bigcup_{p \in Q} \mathfrak{p}$ : if this were not so, then, by (2.1), there would exist $\mathfrak{p}_{0} \in Q$ such that $t s u_{n} \in \mathfrak{p}_{0}$ and $\mathfrak{q}_{1} \subseteq \mathfrak{p}_{0}$, and these relations would imply, respectively, that $\mathfrak{p}_{0} \neq \mathfrak{p}_{1}$ and $\mathfrak{p}_{0}=\mathfrak{p}_{1}$ ! Thus there exist $a \in A$ and $y \in q_{1}$ such that

$$
a t s u_{n}+y \notin \bigcup_{p \in Q} p
$$

thus $u=a t s u_{n}+y$ is such that $\left(u_{1}, \ldots, u_{n-1}, u\right) \in U_{n}$. Note that, by (6) and (1.5),

$$
\operatorname{ann}\left(\frac{t m}{\left(u_{1}, \ldots, u_{n-1}, u\right)}\right)=q_{1} .
$$

Thus, in $U_{n}^{-n} M$,

$$
\begin{aligned}
\frac{t m}{\left(u_{1}, \ldots, u_{n-1}, 1\right)} & =\frac{u t m}{\left(u_{1}, \ldots, u_{n-1}, u\right)}=\frac{\left(a t s u_{n}+y\right) t m}{\left(u_{1}, \ldots, u_{n-1}, u\right)} \\
& =t s u_{n}\left(\frac{a t m}{\left(u_{1}, \ldots, u_{n-1}, u\right)}\right)
\end{aligned}
$$

since $y \in \mathfrak{q}_{1}$. Hence, in $\left(U_{n}^{-n} M\right)_{\mathfrak{p}_{1}}$, by (5),

$$
\delta_{p_{1}}=\frac{a t m}{\frac{\left(u_{1}, \ldots, u_{n-1}, u\right)}{1}}
$$

Moreover, it follows from (7) that

$$
\frac{a t m}{\frac{\left(u_{1}, \ldots, u_{n-1}, u\right)}{1}}=0
$$

in $\left(U_{n}^{-n} M\right)_{p}$ for all $\mathfrak{p} \in \partial F_{n-1} \backslash\left\{p_{1}\right\}$. Thus $\delta=\gamma^{n}\left(\frac{a t m}{\left(u_{1}, \ldots, u_{n-1}, u\right)}\right)$, and the proof is finished.

3. The isomorphism. In this section we shall show that, when $M$ has only finitely many minimal associated primes, then, in the notation of $\$ 2$, the complex $C(\mathcal{U}, M)$ of (2.4) is isomorphic to the Cousin complex $C(\mathscr{F}, M)$. While doing so, we shall achieve rather more, for we shall obtain a characterization of the Cousin complex $C(\mathscr{F}, M)$. It will be convenient to introduce the following definition. 
(3.1) Definition. (Recall that $\mathscr{F}=\left(F_{i}\right)_{i \geq 0}$ is a filtration of $\operatorname{Spec}(A)$ that admits $M$; we make no assumption here about Ass $(M)$.) A complex $X^{\cdot}\left(=\left(X^{i}\right)_{i \geq-2}\right)$ of $A$-modules and $A$-homomorphisms is said to be of Cousin type for $M$ with respect to $\mathscr{F}$ if it has the form

$$
0 \stackrel{d^{-2}}{\longrightarrow} M \stackrel{d^{-1}}{\longrightarrow} X^{0} \longrightarrow \ldots \longrightarrow X^{i} \stackrel{d_{x \cdot}^{i}}{\longrightarrow} X^{i+1} \longrightarrow \ldots
$$

and satisfies the following for each $n \in \mathbb{N}_{0}$ :

(i) $\operatorname{Supp}\left(X^{n}\right) \subseteq F_{n}$;

(ii) $\operatorname{Supp}\left(\operatorname{coker} d_{x}^{n-2}\right) \subseteq F_{n}$;

(iii) $\operatorname{Supp}\left(\operatorname{ker} d_{X}^{n-1} / \operatorname{im} d_{X}^{n-2}\right) \subseteq F_{n+1}$;

(iv) the natural $A$-homomorphism $\xi\left(X^{n}\right): X^{n} \rightarrow \underset{p \in \partial F_{n}}{\bigoplus}\left(X^{n}\right)_{p}$, such that, for $x \in X^{n}$ and $p \in \partial F_{n}$, the component of $\xi\left(X^{n}\right)(x)$ in the summand $\left(X^{n}\right)_{p}$ is $x / 1$ (it follows from condition (i) and [6, (2.2) and (2.3)] that there is such an A-homomorphism), is an isomorphism.

(3.2) Remarks. (i) It follows from (1.1) and (1.2)(ii) that the Cousin complex $C(\mathscr{F}, M)$ is a complex of Cousin type for $M$ with respect to $\mathscr{F}$.

(ii) It follows from (2.5) and (2.6) that, in the case when $M$ has only finitely many minimal associated primes, the complex $C(U, M)$ of (2.4) is of Cousin type for $M$ with respect to $\mathscr{F}$.

Our next result, when taken in conjunction with (3.2)(i), shows, among other things, that every complex of Cousin type for $M$ with respect to $\mathscr{F}$ is isomorphic to the Cousin complex $C(\mathscr{F}, M)$.

(3.3) THEOREM. $\left(\mathscr{F}=\left(F_{i}\right)_{i \geq 0}\right.$ is a filtration of $\operatorname{Spec}(A)$ that admits $M$; we make no assumption here about $\operatorname{Ass}(M)$.) Let $X^{\cdot}=\left(X^{i}\right)_{i \geq-2}$ and $Y^{\cdot}=\left(Y^{i}\right)_{i \geq-2}$ be complexes of Cousin type for $M$ with respect to $\mathscr{F}$. Then there is exactly one morphism of complexes

$$
\Phi=\left(\phi^{i}\right)_{i \geqslant-2}: X^{\cdot} \rightarrow Y^{\cdot}
$$

which is such that $\phi^{-1}: M \rightarrow M$ is the identity mapping; moreover, this morphism is an isomorphism.

Proof. Let $n \in \mathbb{N}_{0}$ and suppose that we have proved that there is exactly one family of $A$-homomorphisms $\left(\phi^{i}\right)_{-2 \leqslant i \leqslant n-1}$ such that

(a) $\phi^{i}: X^{i} \rightarrow Y^{i}$ for each $i=-2, \ldots, n-1$,

(b) $\phi^{-1}: M \rightarrow M$ is the identity map, and

(c) the diagram

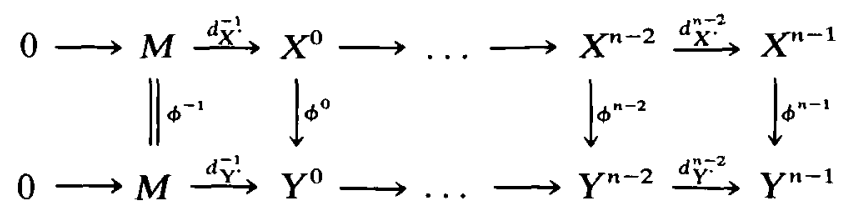

commutes; suppose also that we have proved that this family $\left(\phi^{i}\right)_{-2 \leqslant i \leqslant n-1}$ is such that $\phi^{i}$ must be an isomorphism for each $i=-2, \ldots, n-1$. 
The above is certainly the case when $n=0$.

Let $\mathfrak{p} \in \partial F_{n}$. By (3.1)(i), (ii), (iii), the commutative diagram

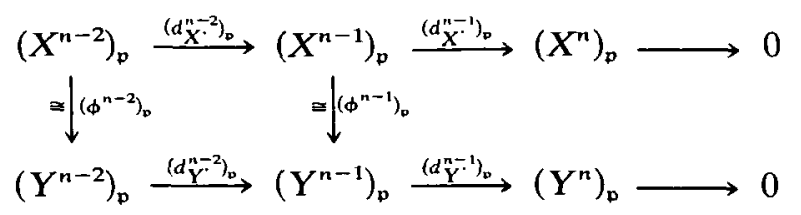

has exact rows. Hence there is exactly one $A_{\mathfrak{p}}$-homomorphism $\mu(n, \mathfrak{p}):\left(X^{n}\right)_{\mathfrak{v}} \rightarrow\left(Y^{n}\right)_{\mathfrak{p}}$ which is such that, when it is inserted in (8), the extended diagram still commutes; moreover $\mu(n, \mathfrak{p})$ is an isomorphism. It thus follows that, if there exists an $A$ homomorphism $\phi^{n}: X^{n} \rightarrow Y^{n}$ such that the diagram

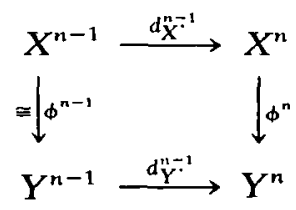

commutes, then $\left(\phi^{n}\right)_{\mathfrak{p}}=\mu(n, \mathfrak{p})$ for each $\mathfrak{p} \in \partial F_{n}$. Moreover, it is clear that such a $\phi^{n}$ must have the property that the diagram

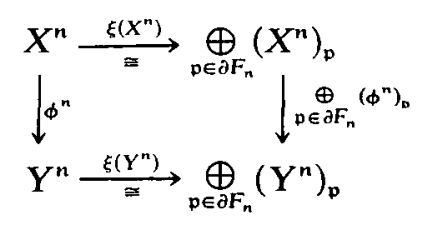

commutes; it therefore follows that there is at most one $A$-homomorphism $\phi^{n}: X^{n} \rightarrow Y^{n}$ such that diagram (9) commutes and that, if one does exist, then it must be an isomorphism.

Now define $\phi^{n}: X^{n} \rightarrow Y^{n}$ to be the unique $A$-homomorphism such that the diagram

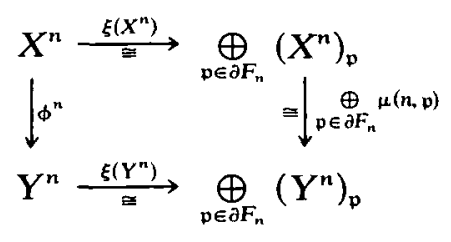

commutes: it is not difficult to see that this $\phi^{n}$ makes the diagram (9) commute.

All the claims in the statement of the theorem now follow from the above inductive argument.

The following corollary is now immediate from (3.3) and (3.2).

(3.4) COROllary. Assume that Ass $(M)$ contains only finitely many minimal members. Then the Cousin complex $C(\mathscr{F}, M)$ is isomorphic to the complex $C(U, M)$ of modules of generalized fractions of (2.4). 
In fact, there is exactly one morphism of complexes

$$
\Phi=\left(\phi^{i}\right)_{i \gg-2}: C(\mathscr{U}, M) \rightarrow C(\mathscr{F}, M)
$$

which is such that $\phi^{-1}: M \rightarrow M$ is the identity mapping, and, moreover, $\Phi$ is an isomorphism.

It should be noted that the hypothesis on $\operatorname{Ass}(M)$ in (3.4) is satisfied not only when $M$ is finitely generated, but also when $A$ is local and $M$ is a balanced big Cohen-Macaulay $A$-module: see [10, (2.4)].

We show next how the isomorphism of complexes $\Phi$ of (3.4) may be described in terms of repeated division.

(3.5) Lemma. Assume that Ass $(M)$ contains only finitely many minimal members. Let $n \in \mathbb{N}$, let $\left(u_{1}, \ldots, u_{n}\right) \in U_{n}$ and let $x \in M$. Then $u_{1}, \ldots, u_{n}$ divides $x$ with respect to $\mathscr{F}$.

Proof. We use induction on $n$; the assertion in the case when $n=1$ is an immediate consequence of the definition of $U_{1}$. Thus we suppose that $n>1$ and the claim has been proved for sequences in $U_{n-1}$. In particular, $x$ is divisible by $u_{1}, \ldots, u_{n-1}$ with respect to F. By $[12,(2.4)(i)]$,

$$
\operatorname{ann}(x)+\sum_{i=1}^{n-1} A u_{i} \subseteq \operatorname{ann}\left(\pi^{n-2}\left(x \div u_{1}, \ldots, u_{n-1}\right)\right) ;
$$

it now follows from the definition of $U_{n}$ that $x$ is divisible by $u_{1}, \ldots, u_{n}$ with respect to $\mathscr{F}_{F}$.

(3.6) ThEOREM. Assume that Ass $(M)$ contains only finitely many minimal members. Then the isomorphism of complexes

$$
\Phi=\left(\phi^{i}\right)_{i \geq-2}: C(\mathcal{U}, M) \rightarrow C(\mathscr{F}, M)
$$

of (3.4) is such that, for each $n \in \mathbb{N}, x \in M$ and $\left(u_{1}, \ldots, u_{n}\right) \in U_{n}$, we have

$$
\phi^{n-1}\left(\frac{x}{\left(u_{1}, \ldots, u_{n}\right)}\right)=x \div u_{1}, \ldots, u_{n} .
$$

Proof. It follows from the commutativity of the diagram

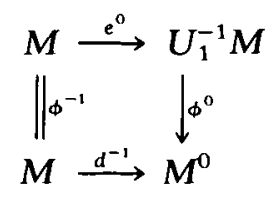

that $u_{1} \phi^{0}\left(\frac{x}{\left(u_{1}\right)}\right)=\phi^{0}\left(e^{0}(x)\right)=d^{-1}(x)=\left(\alpha_{\mathfrak{p}}\right)_{\mathfrak{p} \in \partial F_{0}}$ where $\alpha_{\mathfrak{p}}=x / 1$ for each $\mathfrak{p} \in \partial F_{0}$. Write $\phi^{0}\left(\frac{x}{\left(u_{1}\right)}\right)=\left(\beta_{\mathfrak{p}}\right)_{\mathfrak{p} \in \partial F_{0}}$.

Let $\mathfrak{p}_{0} \in \partial F_{0}$. If $\operatorname{ann}(x) \subseteq \mathfrak{p}_{0}$, then $u_{1} \notin \mathfrak{p}_{0}$ and $u_{1} \beta_{\mathfrak{p}_{0}}=\alpha_{\mathfrak{p}_{0}}$ : it follows that for such a $\mathfrak{p}_{0}$ we 
must have $\beta_{\mathfrak{p}_{0}}=x / u_{1}$. On the other hand, if $\operatorname{ann}(x) \nsubseteq \mathfrak{p}_{0}$, choose $s \in \operatorname{ann}(x) \backslash \mathfrak{p}_{0}$; then

$$
s\left(\beta_{\mathfrak{p}}\right)_{\mathfrak{p} \in \partial F_{0}}=s \phi^{\circ}\left(\frac{x}{\left(u_{1}\right)}\right)=\phi^{0}\left(\frac{s x}{\left(u_{1}\right)}\right)=0,
$$

and it follows that in this case $\beta_{\mathfrak{p}_{0}}=0$. Thus $\phi^{\circ}\left(\frac{x}{\left(u_{1}\right)}\right)=x \div u_{1}$.

Now suppose, inductively, that $n>1$ and that the claim in the statement of the theorem has been proved for sequences in $U_{n-1}$. In particular,

$$
\phi^{n-2}\left(\frac{x}{\left(u_{1}, \ldots, u_{n-1}\right)}\right)=x \div u_{1}, \ldots, u_{n-1} .
$$

Let $z=\pi^{n-2}\left(x \div u_{1}, \ldots, u_{n-1}\right)$ (the notation is as in (1.4)). It follows from the commutativity of the diagram



that

$$
\begin{aligned}
u_{n} \phi^{n-1}\left(\frac{x}{\left(u_{1}, \ldots, u_{n}\right)}\right) & =\phi^{n-1} \circ e^{n-1}\left(\frac{x}{\left(u_{1}, \ldots, u_{n-1}\right)}\right)=d^{n-2}\left(x \div u_{1}, \ldots, u_{n-1}\right) \\
& =\left(\alpha_{p}\right)_{p \in \partial F_{n-1}}
\end{aligned}
$$

where $\alpha_{\mathfrak{p}}=z / 1$ for each $\mathfrak{p} \in \partial F_{n-1}$. Write $\phi^{n-1}\left(\frac{x}{\left(u_{1}, \ldots, u_{n}\right)}\right)=\left(\beta_{\mathfrak{p}}\right)_{\mathfrak{p} \in \partial F_{n-1}}$.

Let $\mathfrak{p}_{n-1} \in \partial F_{n-1}$. If $\operatorname{ann}(z) \subseteq \mathfrak{p}_{n-1}$, then $u_{n} \notin \mathfrak{p}_{n-1}$ by (3.5); also $u_{n} \beta_{\mathfrak{p}_{n-1}}=\alpha_{\mathfrak{p}_{n-1}}=\frac{z}{1}$, and so $\beta_{\mathfrak{p}_{n-1}}=\frac{z}{u_{n}}$ in this case.

Now consider the remaining case, where $\operatorname{ann}(z) \nsubseteq \mathfrak{p}_{n-1}$. Choose $t \in \operatorname{ann}(z) \backslash \mathfrak{p}_{n-1}$. It follows from the facts that $\Phi$ is an isomorphism of complexes and

$$
\phi^{n-2}\left(\frac{x}{\left(u_{1}, \ldots, u_{n-1}\right)}\right)=x \div u_{1}, \ldots, u_{n-1} \text { that } \frac{t x}{\left(u_{1}, \ldots, u_{n-1}\right)} \in \operatorname{im} e^{n-2}
$$

hence, on use of (1.5), it follows that

$$
\frac{t x}{\left(u_{1}, \ldots, u_{n-1}, u_{n}\right)}=0
$$

in $U_{n}^{-n} M$. Now apply $\phi^{n-1}$ to this to see that $t \beta_{\mathfrak{p}_{n-1}}=0$; thus in this case $\beta_{\mathfrak{p}_{n-1}}=0$. It follows that

as required.

$$
\phi^{n-1}\left(\frac{x}{\left(u_{1}, \ldots, u_{n}\right)}\right)=x \div u_{1}, \ldots, u_{n}
$$


Let us now indicate how the two results of Zakeri mentioned in the introduction can be deduced from Theorems (3.4) and (3.6).

(3.7) EXAMPLE. Consider the special case in which $M$ is a non-zero finitely generated Cohen-Macaulay $A$-module, and $\mathscr{F}=\mathscr{H}(M)$, the $M$-height filtration of $\operatorname{Spec}(A)$. Then the hypotheses of Theorems (3.4) and (3.6) are satisfied. It is not difficult to see that, in this case,

$$
U_{i}=\left\{\left(u_{1}, \ldots, u_{i}\right) \in A^{i}: u_{1}, \ldots, u_{i} \text { is a poor } M \text {-sequence }\right\}
$$

for each $i \in \mathbb{N}$. We obtain from (3.4) and (3.6) an isomorphism of complexes

$$
\Phi=\left(\phi^{i}\right)_{i \geqslant-2}: C(U, M) \rightarrow C(\mathscr{H}(M), M)=C(M)
$$

which is such that $\phi^{-1}$ is the identity map on $M$ and, for each $n \in \mathbb{N}, x \in M$ and poor $M$-sequence $\left(u_{1}, \ldots, u_{n}\right)$, we have

$$
\phi^{n-1}\left(\frac{x}{\left(u_{1}, \ldots, u_{n}\right)}\right)=x \div u_{1}, \ldots, u_{n} .
$$

This result was first obtained by Zakeri: see [15, Chapter III, Theorem (3.7)].

(3.8) Example. Now consider the special case in which $A$ is local, with maximal ideal $\mathrm{m}$, and $M$ is a balanced big Cohen-Macaulay $A$-module. Take for $\mathscr{F}$ the dimension filtration $\mathscr{D}(A)$ of $\operatorname{Spec}(A)$. By [10, (2.4)], the hypotheses of (3.4) and (3.6) are satisfied. This time,

$$
U_{n}=\left\{\left(u_{1}, \ldots, u_{n}\right) \in A^{n}: \text { for each } i=1, \ldots, n, \sum_{i=1}^{i} A u_{j} \nsubseteq \mathfrak{p}\right.
$$

for all $\mathfrak{p} \in \operatorname{Supp}(M)$ for which $\operatorname{dim} A / \mathfrak{p}=\operatorname{dim} A-i+1\}$

for each $n \in \mathbb{N}$. It follows from [10, (2.4)] that each $U_{n}$ consists of poor $M$-sequences. Note also that, if $1 \leqslant i \leqslant \operatorname{dim} A$ and $s_{1}, \ldots, s_{i}$ form a subset of a system of parameters for $A$, then $\left(s_{1}, \ldots, s_{i}\right) \in U_{i}$.

From (3.4) and (3.6) we obtain an isomorphism of complexes

$$
\Phi: C(\mathcal{U}, M) \rightarrow C(\mathscr{D}(A), M) .
$$

However, in [15, Chapter IV, Theorem (3.15)] Zakeri obtained an apparently different result. For each $i \in \mathbb{N}$, let

$$
T_{i}=\left\{\left(t_{1}, \ldots, t_{i}\right) \in A^{i}: t_{1}, \ldots, t_{i} \text { is a poor } M \text {-sequence }\right\} .
$$

It is not difficult to use $[13,(3.10)]$ and $\left[10,(2.5),(2.7)\right.$ and (2.8)] to see that $T_{i}$ is a triangular subset of $A^{i}$ for each $i \in \mathbb{N}$. In fact, $\mathscr{T}=\left(T_{i}\right)_{i \in \mathbb{N}}$ is a chain of triangular subsets on $A$, so that the complex $C(\mathscr{T}, M)$ may be formed as explained in the introduction.

By $[11,3.7], C(\mathscr{D}(A), M)$ is exact, so that by $[12,(4.3)]$ each poor $M$-sequence divides each element of $M$ with respect to $\mathscr{D}(A)$. In [15, Chapter IV, Theorem (3.15)], Zakeri established the existence of an isomorphism of complexes

$$
\Psi=\left(\psi^{i}\right)_{i \geq-2}: C(\mathscr{T}, M) \rightarrow C(\mathscr{D}(A), M)
$$


ADRIAN M. RILEY, RODNEY Y. SHARP AND HOSSEIN ZAKERI

which is such that $\psi^{-1}$ is the identity map on $M$ and, for each $n \in \mathbb{N}, x \in M$ and $\left(t_{1}, \ldots, t_{n}\right) \in T_{n}$, we have

$$
\psi^{n-1}\left(\frac{x}{\left(t_{1}, \ldots, t_{n}\right)}\right)=x \div t_{1}, \ldots, t_{n} .
$$

In fact, it is possible to obtain Zakeri's isomorphism from our isomorphism $\Phi$ of (3.4) and (3.6) by use of the following lemma, which can itself be proved by an extension of the ideas of the proof of $[\mathbf{1 0},(2.2)]$. We omit the details; the interested reader will find [14, 3.15] helpful.

(3.9) Lemma. Let $A$ be local and let $M$ be a balanced big Cohen-Macaulay Amodule. Then for each $M$-sequence $x_{1}, \ldots, x_{r}$ there exist $b_{1}, \ldots, b_{r} \in A$ such that $x_{1}+b_{1}, \ldots, x_{r}+b_{r}$ form $a$ subset of a system of parameters for $A$ and

$$
b_{i} M \subseteq\left(\sum_{j=1}^{i-1} A x_{j}\right) M
$$

for all $i=1, \ldots, r$.

Our final result, which is immediate from (3.6), is a generalization of part of Theorem (4.8) of [12], the very result that led to the discovery of modules of generalized fractions!

(3.10) Corollary (of (3.4) and (3.6)). Suppose that Ass(M) contains only finitely many minimal members. Let $q \in \mathbb{N}_{0}$ and let $\beta \in M^{q}$. Then there exists $y \in M$ and $\left(u_{1}, \ldots, u_{q+1}\right) \in U_{q+1}$ such that $\beta=m \div u_{1}, \ldots, u_{q+1}$.

(3.11) Concluding Remarks. Although modules of generalized fractions are relatively new, Cousin complexes have numerous interactions with topics that frequently occur in the literature on commutative algebra. Not only can they be used to characterize Cohen-Macaulay rings (as was mentioned in the introduction) and Gorenstein rings, but they also reflect some of the structure of the minimal injective resolution of a Gorenstein ring that is brought out in Bass's Fundamental Theorem [1]: see [6, (5.5)]. Their use in the theory of Gorenstein modules [7] means that they have connections with the canonical modules of Herzog and Kunz [3]. They can be used to characterize the (commutative Noetherian) rings that satisfy the conditions $\left(S_{k}\right)[\mathbf{5}$, p. 125]: see [8]. They have connections with the local cohomology theory of Grothendieck and Hartshorne [2]: see [9]. And, as was mentioned in the introduction, they may be used to characterize balanced big Cohen-Macaulay modules: the relevance of big Cohen-Macaulay modules to the homological conjectures in local algebra is explained in [4].

It is hoped that the results of this paper will make Cousin complexes easier to work with; in any event, we believe that the relationships between Cousin complexes and modules of generalized fractions established in this paper, and the above connections between Cousin complexes and other topics in commutative algebra, provide evidence to support our view that modules of generalized fractions are worth further investigation. 


\section{REFERENCES}

1. H. Bass, On the ubiquity of Gorenstein rings, Math. Z. 82 (1963), 8-28. 1967).

2. A. Grothendieck, Local cohomology, Lecture Notes in Mathematics No. 41 (Springer,

3. J. Herzog and E. Kunz, Der kanonische Modul eines Cohen-Macaulay Rings, Lecture Notes in Mathematics No. 238 (Springer, 1971).

4. M. Hochster, Topics in the homological theory of modules over commutative rings, C.B.M.S. Regional Conference Series in Mathematics No. 24 (American Mathematical Society, 1975).

5. H. Matsumura, Commutative algebra (Benjamin, 1980).

6. R. Y. Sharp, The Cousin complex for a module over a commutative Noetherian ring, Math Z. 112 (1969), 340-356.

7. R. Y. Sharp, Gorenstein modules, Math. Z. 115 (1970), 117-139.

8. R. Y. Sharp, Cousin complex characterizations of two classes of commutative Noetherian rings, J. London Math. Soc. (2) 3 (1971), 621-624.

9. R. Y. Sharp, Local cohomology and the Cousin complex for a commutative Noetherian ring, Math. Z. 153 (1977), 19-22.

10. R. Y. Sharp, Cohen-Macaulay properties for balanced big Cohen-Macaulay modules, Math. Proc. Cambridge Philos. Soc. 90 (1981), 229-238.

11. R. Y. Sharp, A Cousin complex characterization of balanced big Cohen-Macaulay modules, Quart. J. Math. Oxford Ser. (2) 33 (1982), 471-485.

12. R. Y. Sharp, On the structure of certain exact Cousin complexes, Commutative algebra: proceedings of the Trento conference, Lecture Notes in Pure and Applied Mathematics No. 84 (Marcel Dekker, 1983), 275-290.

13. R. Y. Sharp and H. Zakeri, Modules of generalized fractions, Mathematika 29 (1982), 32-41.

14. R. Y. Sharp and H. Zakeri, Modules of generalized fractions and balanced big CohenMacaulay modules, Commutative algebra: Durham 1981, London Mathematical Society Lecture Notes No. 72 (Cambridge University Press, 1982), 61-82.

15. $\mathrm{H}$. Zakeri, Modules of generalized fractions and their application in commutative algebra, Ph.D. Thesis, University of Sheffield (1982).

Adrian M. Riley and Rodney Y. Sharp

Department of Pure Mathematics

THE UNIVERSITY OF SHEFFIELD

HiCKS BUILDING

SHEFFIELD S3 7RH

ENGLAND
Hossein Zakeri

DEPARTMENT OF MATHEMATICS

UNIVERSITY FOR TEACHER EDUCATION

49 Mobarezan Avenue

TEHRAN

IRAN 